\title{
Theoretical studies on the Creation of Artificial Magnetic Monopoles
}

\author{
Shinichi Ishiguri, Ph.D. \\ Nihon University \\ 1-2-1 Izumi-Cho, Narashinoshi, Chiba 275-8575 JAPAN \\ Email: shinichi.ishiguri@gmail.com
}

\begin{abstract}
The purpose of this paper is to demonstrate the existence of an artificial magnetic monopole and to introduce new electromagnetic equations by altering an electric field and a magnetic field vectors.

As a principle device, a cylindrical condenser is prepared, and a superconducting loop is inserted into it. By this conduction, radial electric fields take a role as the centripetal force and both counterclockwise and clockwise motions are induced. As a result, a stationery wave is formed in which the nodes take a part in creating a monopole as follows.

First, employing the Lorentz conservations and because node of the stationary wave has no phases, the momentum $\boldsymbol{k}$ and the vector potential $\boldsymbol{A}$ vanish and instead a magnetic potential appears in order to maintain the Lorentz conservation. This magnetic potential has relationship with an electric potential, and thus consequently, a dependent relationship is obtained between an electric field and a magnetic field vectors. Using this conclusive dependent relationship, we can derive new Maxwell equation assembly which are created by altering the electric field and the magnetic field vectors. In this process, we derive a divergent equation of magnetic fields which is not zero, i.e., the existence of a magnetic monopole. Employing these newly derived Maxwell equation, an electromagnetic wave is derived whose speed is the same as one the existing Maxwell equations provide. As a monopole configuration, this paper discusses the energy gap of the vacuum, which is a result of the Dirac equation and describes a monopole as pairs between two Cooper pairs (i.e. four electrons) whose interaction is a photon. As mentioned, because the total momenta and phases are zero, this paper defines the wave function as the Dirac function and demonstrate the condensation, employing the Bloch's theorem. Moreover, using the macroscopic basic equations, we retrace the creation of the divergent magnetic field in view of macroscopic phenomenon, which provides results in this paper.

In Result section in this paper, we succeeded in demonstrating the distribution of the divergent magnetic field of monopole in terms of both microscopic and macroscopic scales. Furthermore, Discussion section describes properties a magnetic monopole should follow.
\end{abstract}

\section{Keywords:}

Artificial magnetic monopole, new electromagnetic equations, superconducting loop, stationary wave, conservation of momentum, Lorentz conservation, magnetic potential, Dirac equation, energy gap in vacuum, pair of two Cooper pairs, 


\section{Introduction}

In this study, we discuss the artificial creation of a magnetic monopole and new electromagnetic equations under a specific condition. The first description of a monopole was presented by Dirac [1]. Based on his suggestions, some experiments were conducted [2,3]. The magnetic monopole in spin ice [4,5] and other systems [6-8] have also been discussed. Recently, by employing the concepts of spin and Bose-Einstein condensation, a potential artificial magnetic monopole was created $[9,10]$. Moreover, the emergent magnetic monopoles were reported [14], and the quantum hall effect [13] is related to create magnetic monopoles [15].

In this paper, we propose another method for creating a magnetic monopole and present its theoretical basis, which includes new equations of the relationship between electric and magnetic fields regarding both static and time-dependent fields.

Dirac's paper described the monopole using the gauge. However, as long as the spatially dependent vector potential $\boldsymbol{A}$ is not zero, the monopole cannot be defined because a nonzero $\boldsymbol{A}$ results in the existing Maxwell's second equation, whereby a nonzero $\boldsymbol{A}$ eventually leads to a loop magnetic flux. By employing Lorentz conservations, in this paper, however, radial electrostatic fields and a stationary wave are derived along a superconducting loop, and thus we demonstrate that Maxwell's second equation can be modified, such that the divergence of the magnetic field is not zero.

Recently the monopole-creation method implemented by replacing the anti-Helmholtz coils with larger coils carrying parallel currents [16]. However, because the vector potential $\boldsymbol{A}$ is employed, new electromagnetic equations are not presented. Indeed, the Maxwell equations should be symmetric for an electric field and a magnetic field vectors. In short, many magnetic monopole discussions have been exited, but the new electromagnetic equations, which explain the symmetry for electric and magnetic fields, are not yet reported.

In the cylindrical coordinates, in this paper, a condenser generates electrostatic fields along the radial direction to a superconducting loop. Given these conditions, we discuss the nodes in the stationary wave along the superconducting loop and consider the Lorentz conservations. In this process, the conserved energy is converted into new form that is associated with a magnetic field potential to yield and hold the Lorentz conservations. As a result, we obtain the specific vectors' dependent relationship between electric and magnetic fields. Because this is a dependent relationship, Maxwell's first equation becomes a modified and proposed Maxwell's second equation, which implies the divergent of a magnetic field $\boldsymbol{B}$ is not zero.

We then derived new electromagnetism equations assembly, which describe the exchange of the distribution characteristics of the electric and magnetic fields. Herein the exchanged Maxwell equations' assembly including time-dependent equations are derived. Using these equations, we obtain the electromagnetic wave whose speed is $c\left(=3.0 \times 10^{8} \mathrm{~m} / \mathrm{s}\right)$. Moreover, as the result of consideration, 
the magnetic monopole is found to be the combination between Cooper pair and Cooper pair. Furthermore, this paper determines the wave function that describes a monopole and the state of the condensation of monopoles. Finally, we numerically analyze the magnetic field distribution from monopole. The results demonstrate the divergence of the distribution of the magnetic fields. The significance of the paper is that it obtained theoretically new equations of both electric and magnetic fields under a specific condition. Moreover, this paper presents easier method to gain an artificial magnetic monopole.

\section{Principle}

Fig. 1 shows the schematic of a superconducting loop and cylindrical condenser setup. In this study, the poles of the cylindrical condenser are charged in advance. Using the abovementioned method, we can apply the radial static electric fields to the superconducting loop. Because of these electric fields (i.e., centripetal forces), Cooper pairs move along the loop both clockwise and counterclockwise, and eventually form a stationary wave. This is because, due to the conservation of the total momenta, rotations must be both clockwise and counterclockwise. As mentioned later, this conservation of the total momenta still continues during and after the transition to the monopole phase.

As will be discussed later, creation of a monopole requires nodes along the superconducting stationary wave, and Cooper pairs in this node constitutes the monopole.

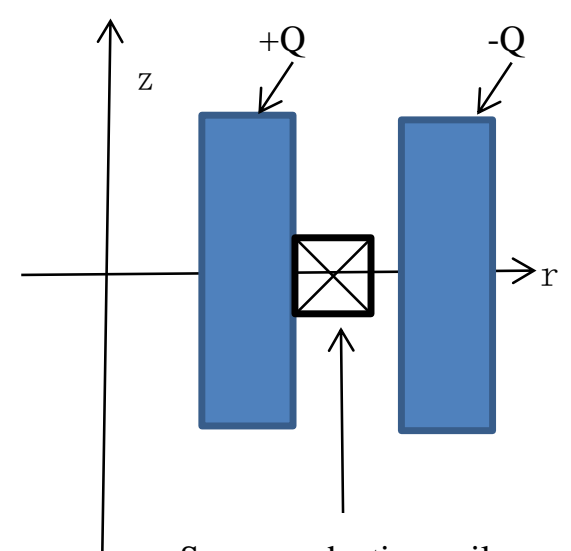

Superconducting coil

Fig. 1 Cross section of the device to create magnetic monopole

\section{Theory}

\subsection{Derivation of the conclusive equation}

As discussed in Principle section, directions of superconducting currents are formed as 
counterclockwise and clockwise rotations. This is because initially the total momenta are zero and thus the conservation of the momentum gives rise to both directions of the superconducting currents. Note that the applied electric field along the radial direction in Principle section works as a centripetal force.

Considering these facts, we can see a stationary wave form along the coil loop. According to [17], solving Schrödinger equation results in the following wave function:

$\psi_{n}=\sqrt{\frac{2}{l}} \sin \left(\frac{n \pi}{l} x\right)$

where

$\psi(x+l)=\psi(x)$

and $\mathrm{n}$ is an integer.

At this point of an integer $\mathrm{n}$, the following Lorentz conservations are considered.

$(\hbar k)^{2}-\left(\frac{\varepsilon}{c}\right)^{2}=$ const.

And

$|\vec{A}|^{2}-\left(\frac{\varphi_{E}}{c}\right)^{2}=$ const.

where

$\mathrm{k}, \varepsilon, \mathrm{c}, \boldsymbol{A}$ and $\varphi_{\mathrm{E}}$ denote the wave number, the energy, speed of light, vector potential and electric potential, respectively.

Importantly each node along the stationary wave has no momentum because the nodes have no phases, assuming that each rotation results in a plane wave.

This implies that

$\hbar k \rightarrow 0$.

The above equation also implies that

$\mathrm{A} \rightarrow 0$.

However, in order to conserve the Lorentz conservation, if the first terms become zero, then the Lorentz conservations do not form in terms of mathematic equations. More concretely, the following equation is considered.

$|\vec{A}|^{2}-\left(\frac{\varphi_{E}}{c}\right)^{2}=\left(\frac{1}{c e} \frac{1}{2} \hbar \omega\right)^{2}$,

where e and $\omega$ denote the charge of an electron and angular frequency, respectively.

When the first term $\boldsymbol{A}$ disappears, the right-hand side is consumed to maintain the mathematical meaning and the Lorentz conservation. As a result, the first term reappears as a different form from the vector potential $\boldsymbol{A}$. Thus, instead of the vector potential $\boldsymbol{A}$, a scalar potential $\varphi_{\mathrm{H}}$ appears: 
$\left(\mu_{0} \varphi_{H}\right)^{2}-\left(\frac{\varphi_{E}}{c}\right)^{2} \equiv 0$

That is,

$\varphi_{E}=c \mu_{0} \varphi_{H}$,

where $\mu_{0}$ denotes the magnetic permeability in the vacuum.

Considering dimension of the above equation, the scalar potential $\varphi_{\mathrm{H}}$ has the unit of a magnetic potential. Therefore, the following conclusive equation is derived.

$\vec{E}=c \vec{B}$

where

$\vec{B}=\mu_{0} \vec{H}$.

This relation is substituted to the existing Gauss equation:

$\operatorname{div} \vec{B}=\frac{\rho}{\varepsilon_{0} c} \neq 0$

where $\rho$ and $\varepsilon_{0}$ denote the charge density and the permittivity in the vacuum.

The above equation (11) does not already imply the existing Gauss equation relationship regarding the electric fields; hence, the distribution of the electric fields must be approached in another way. Because Eq. (11) is not consistent with the existing Maxwell's second equation, the following equation must be derived from Eq. (9):

$\operatorname{div} \vec{E}=0$.

Eqs. (11) and (12) imply that the distribution characteristics of the electric and magnetic fields have been exchanged. We know that the looped magnetic flux is generally distributed when a current is supplied along a coil. In this case, however, we can infer that the looped electric flux is created; combining the $\mu_{0}$ and $c$, a magnetic current can be considered in this case. Because the rotation of the electric field is not zero, we can consider a modified Ampere's law, using the conclusive equation (9) $\operatorname{rot} \vec{E}=\mu_{0} c \vec{\imath} \rightarrow \vec{\imath}_{B}$.

The right-hand side is the magnetic current density $\boldsymbol{i}_{\mathrm{B}}$.

In Table 1, the differences are shown between the existing and proposed Maxwell equations regarding static fields.

Table 1 Summary of the differences between the existing equations and new equations in terms of static fields

\begin{tabular}{|c|c|}
\hline Existing Maxwell's equation & Proposed Maxwell's equation \\
\hline $\operatorname{div} \vec{E}=\frac{\rho}{\varepsilon_{0}}$ & $\operatorname{div} \vec{E}=0$ \\
\hline $\operatorname{div} \vec{B}=0$ & $\operatorname{div} \vec{B}=\frac{\rho}{\varepsilon_{0} c}$ \\
\hline $\operatorname{rot} \vec{E}=0$ & $\operatorname{rot} \vec{E}=\mu_{0} c \vec{\imath}$ \\
\hline $\operatorname{rot} \vec{H}=\vec{\imath}$ & $\operatorname{rot} \vec{H}=0$ \\
\hline
\end{tabular}




\subsection{Derivation of time-dependent equations and electromagnetic wave}

Let us consider time-dependent Maxwell equations and the derivation of an electromagnetic wave. That is, to demonstrate that our derived time-dependent Maxwell equations are valid, it is imperative to derive the electromagnetic wave from them.

As everyone knows, the existing time-dependent Maxwell equations are

$$
\operatorname{rot} \vec{H}=\frac{\partial \vec{D}}{\partial t}
$$

and

$$
\operatorname{rot} \vec{E}=-\frac{\partial \vec{B}}{\partial t}
$$

where $\boldsymbol{H}$ and $\boldsymbol{D}$ denote the magnitude of a magnetic field and the electric flux density, respectively. To the above two equations, the following exchanges are made to be subjected according to the conclusive equation (9).

$\vec{E} \rightarrow c \vec{B}$

And

$\vec{B} \rightarrow \frac{\vec{E}}{c}$

Thus the third equation a monopole follows becomes

$\operatorname{rot} \vec{E}=c^{2} \varepsilon_{0} \mu_{0} \frac{\partial \vec{B}}{\partial t}$ (17) for a monopole

Then the firth equation a monopole follows are derived as

$\operatorname{rot} \vec{B}=-\frac{1}{c^{2}} \frac{\partial \vec{E}}{\partial t} . \quad$ (18) for a monopole

Next let us derive a wave equation to electromagnetic wave from the above-mentioned two equations a monopole holds.

Taking a rotation-operator for the both sides of Eq. (17),

$\operatorname{rot}(\operatorname{rot} \vec{E})=c^{2} \varepsilon_{0} \mu_{0} \frac{\partial}{\partial t}(\operatorname{rot} \vec{B})$

To this equation, Eq. (18) is substituted.

$\operatorname{rot}(\operatorname{rot} \vec{E})=-\varepsilon_{0} \mu_{0} \frac{\partial^{2} \vec{E}}{\partial t^{2}}$

From the vector analysis formula, a wave equation for electric fields is derived:

$\nabla^{2} \vec{E}=\varepsilon_{0} \mu_{0} \frac{\partial^{2} \vec{E}}{\partial t^{2}}$ 
where

$\nabla \cdot \vec{E} \equiv 0$

Thus velocity $v$ of the wave equation becomes

$\mathrm{v}=\frac{1}{\sqrt{\varepsilon_{0} \mu_{0}}} \rightarrow c$

Similarly, a wave equation for magnetic fields can be derived by the dual calculations;

$\frac{\partial^{2} \vec{B}}{\partial t^{2}}=\frac{1}{\varepsilon_{0} \mu_{0}} \nabla^{2} \vec{B}$

Thus velocity for the wave equation of magnetic fields is obtained as the same form.

$\mathrm{v}=\frac{1}{\sqrt{\varepsilon_{0} \mu_{0}}} \rightarrow c$

In Table 2, the differences of time-dependent Maxwell equations are summarized.

Table 2 summary of time-dependent Maxwell equations

\begin{tabular}{|c|c|}
\hline Existing equation & The proposed equation \\
\hline $\operatorname{rot} \vec{E}=-\frac{\partial \vec{B}}{\partial t}$ & $\operatorname{rot} \vec{E}=c^{2} \varepsilon_{0} \mu_{0} \frac{\partial \vec{B}}{\partial t}$ \\
\hline $\operatorname{rot} \vec{H}=\frac{\partial \vec{D}}{\partial t}$ & $\operatorname{rot} \vec{B}=-\frac{1}{c^{2}} \frac{\partial \vec{E}}{\partial t}$ \\
\hline
\end{tabular}

\subsection{Structure of a monopole}

As discussed in the previous section 3.1, in the process of the conservation of the Lorentz equation (42) and to create a monopole, the photon $\hbar \omega\left(=2 m c^{2}\right)$ which is defined by the Dirac equation must be consumed. Note that right-hand side of Eq. (4-2) contains the photon energy $\hbar \omega$. Considering the stationary wave derived from both the clockwise and the counterclockwise waves, it can be assumed that 2 Cooper pairs (i.e., 4 electrons) gather at each node. At each node, as indicated in Fig. 2, a Cooper pair shifts from the energy level of the vacuum, while maintaining the pair, to the ground state. At this point, we can derive the following equation:

$2 m c^{2}=\hbar \omega$

where $m$ and $\omega$ denote the electron mass and the angular frequency, respectively. 


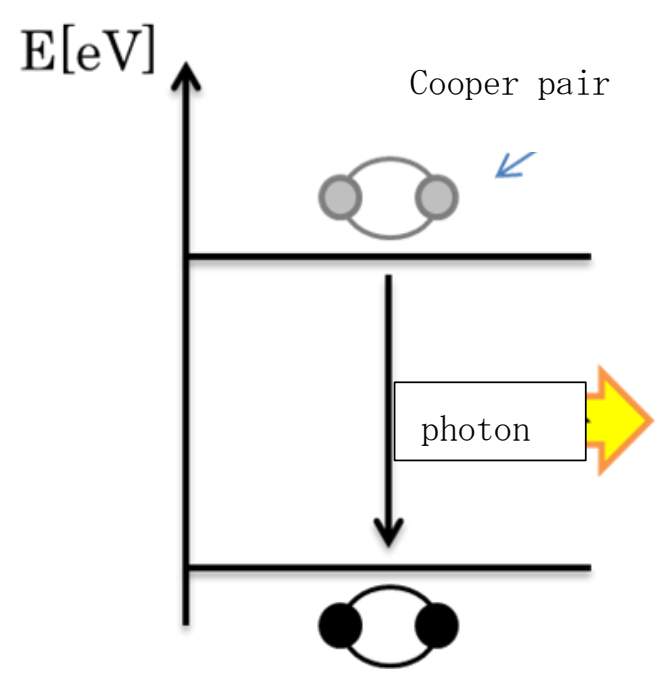

Fig. 2 Transition of a Cooper pair between the energy gap of vacuum

As shown in Fig. 3, when Cooper pair 1 shifts to the ground state, a photon is emitted. This photon works into Cooper pair 2, which is located at the same node as Cooper pair 1, and which is at the ground state and the energy level of this Cooper pair increases to the energy level of the vacuum. In turn, Cooper pair 2 shifts from the energy level of the vacuum to the ground state, and a photon is again emitted. Similarly, this photon works into Cooper pair 1 and the energy of this pair increases to the energy level of the vacuum. In other words, at a node, the two Cooper pairs undergo exchanging interaction in terms of a photon. In this sense, a monopole is a multiple particle with photons acting as an interaction force. We estimate the interaction as follows:

$\hbar \omega=2 m c^{2}=1.64 \times 10^{-13}[J]$.

The energy gap $\Delta E$ and time period $\Delta t$ have an uncertain relation in this case. This fact provides some basis for the extremely small radius of the monopole. As a result of the transition to the ground state of the Cooper pair, this Cooper pair decreases its energy. This implies that the coherence of the Cooper pair becomes substantially shorter. That is, the pair is not broken but takes stronger combination energy among it. Consequently, the magnetic monopole is the combination between Cooper pair and Cooper pair. 


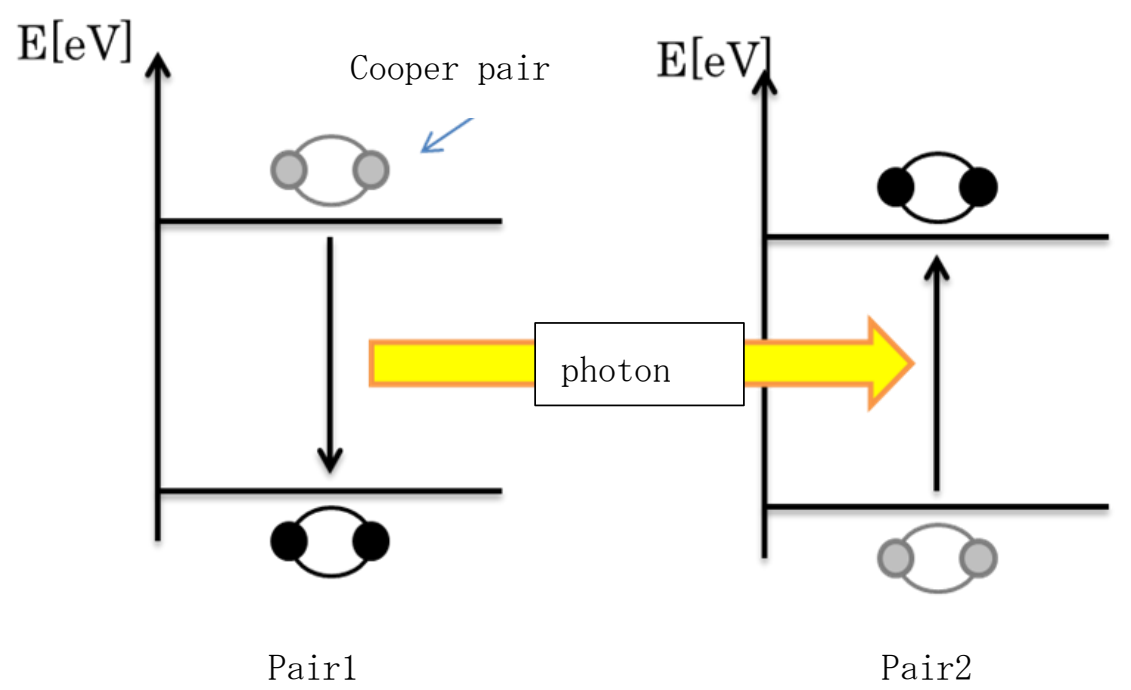

Fig. 3 Schematic that photon works to a Cooper pair in the vicinity of another Cooper. A photon is exchanged between Pair 1 and Pair 2.

\subsection{Condensation of wave functions of monopoles}

It is assumed that both before and after the transition to monopole phase, the total momenta should be conserved. Before the transition, because the motions of Cooper pairs have both clockwise and counterclockwise rotations, the total momenta are zero. It is also assumed that each wave function along each rotation is approximated as a plane wave which behaves as a boson. Considering both wave functions as simple product and after the transition, therefore, the net wave function at each node can be derived as

$\left|\psi_{i}(r)\right|^{2} \equiv \delta(r)$

where the normalization is satisfied as

$\int\left\lceil\psi_{i}\right\rceil^{2} d v=1$.

From the Bloch's theorem,

$\psi_{i}\left(\mathrm{r}+R_{i}\right)=\exp \left(j k R_{i}\right) \psi_{i}$,

where $\mathrm{j}$ and $\mathrm{k}$ denote the imagery unit and a wave number, respectively.

Thus the total wave function $\Phi$ results in

$\Phi=\prod^{i} \psi_{i}\left(r+R_{i}\right)=\exp \left(j k \sum^{i} R_{i}\right)\left[\psi_{i}\right]^{n}=\exp \left(\mathrm{j} \sum^{i} k R_{i}\right)\left[\psi_{i}\right]^{n}$.

In this equation, the wavenumber $k$ is defined as

$\mathrm{k}=\alpha\left(\frac{\pi}{R_{i}}\right)$

where $\pi / \mathrm{R}_{\mathrm{i}}$ denote the Brillouin zone

Therefore, the above total wave function is calculated as 


$$
\Phi=\exp (j \pi n \alpha)\left[\psi_{i}\right]^{n} .
$$

Considering the property of a general phase, $\alpha \equiv 2$ must be held.

At this time, the total wave function becomes conclusively

$\Phi=\left[\psi_{i}\right]^{n}=\left[\psi_{i}\right]^{2 M}=\delta^{M}$.

Then consider the normalization of the total wave function;

$\int|\Phi|^{2} d V=\int|\Phi|^{2} d v_{1} d v_{2} \cdots d v_{M}=\int \delta^{M} d v_{1} d v_{2} \cdots d v_{M}$.

On the other hand, and as mentioned, the normalization of an element wave function is

$\int \delta^{i} d v_{i}=1$.

Accordingly,

$\int|\Phi|^{2} d V=1$.

\subsection{Preparation of simulating equations}

This section now considers simulating and macroscopic equations. Let us consider simulating equations in view of retraced calculations.

From a Lorentz conservation,

$p^{2}-\left(\frac{e \varphi_{E}}{c}\right)^{2}=0$,

where $p$ denotes a macroscopic momentum.

That is,

$p= \pm \frac{e \varphi_{E}}{c}$.

If

$p=m v$,

then

$v= \pm \frac{e \varphi_{E}}{m c}$.

Thus, a current density is

$j=\mathrm{e} n_{s} v= \pm e n_{s} \frac{e \varphi_{E}}{m c}$,

where $n_{\mathrm{s}}$ is the concentration of Cooper pairs that can be derived as

$n_{s}=\frac{1}{\xi^{3}}$,

where $\xi$ denotes the coherence of a Cooper pair.

From the existing Maxwell equation,

$\frac{\partial B_{Z}}{\partial R}=\mu_{0} j$.

Thus

$\frac{\partial B_{Z}}{\partial R}= \pm \mu_{0} n_{s} e^{2} \frac{\varphi_{E}}{m c}$ 
Consequently, the magnetic field components along z-direction becomes $B_{z}= \pm \mu_{0} n_{s} e^{2} \frac{\varphi_{E}}{m c} R$,

where $R$ denotes a radius of coil in the proposed device (see section 2).

Next let us consider the equations of the divergence of magnetic field. First, the relationship between an electric potential and an electric field is simply

$\varphi_{E}=-\int E_{z} d z$.

This equation is substituted to the abovementioned equation (45) of magnetic field component.

$B_{z}=\mp \mu_{0} n_{s} e^{2} \frac{R}{m c} \int E_{z} d z$.

Differentials are taken to both sides, and thus

$\mathrm{d} B_{Z}=\mp \mu_{0} n_{s} e^{2} \frac{R}{m c} E_{Z} d z$.

Therefore

$\operatorname{div} \vec{B} \equiv \frac{d B_{z}}{d z}=\mp \mu_{0} n_{s} e^{2} \frac{R}{m c} E_{z}$.

To implement of this equation, it is necessary to obtain the electric field component $E_{\mathrm{z}}$.

The Poisson's equation is now considered.

$\frac{d^{2} \varphi_{E}}{d z^{2}}=-\frac{2 e n_{s}}{\varepsilon_{0}}$.

Thus

$-E_{z}=-\frac{2 e n_{s}}{\varepsilon_{0}} Z$

Here $z$ is assumed to be the coherence $\xi$.

Thus

$E_{z}=\frac{2 e n_{s}}{\varepsilon_{0}} \xi$

Consequently,

$\operatorname{div} \vec{B}=\mp \mu_{0} n_{s} e^{2} \frac{R}{m c}\left(\frac{2 e n_{s}}{\varepsilon_{0}} \xi\right)$.

\section{Result}

Fig. 4 and Fig. 5 show the microscopic picture of a monopole. These figures were created from the potential equation in the electromagnetism, employing the conclusive Eq. (9) 


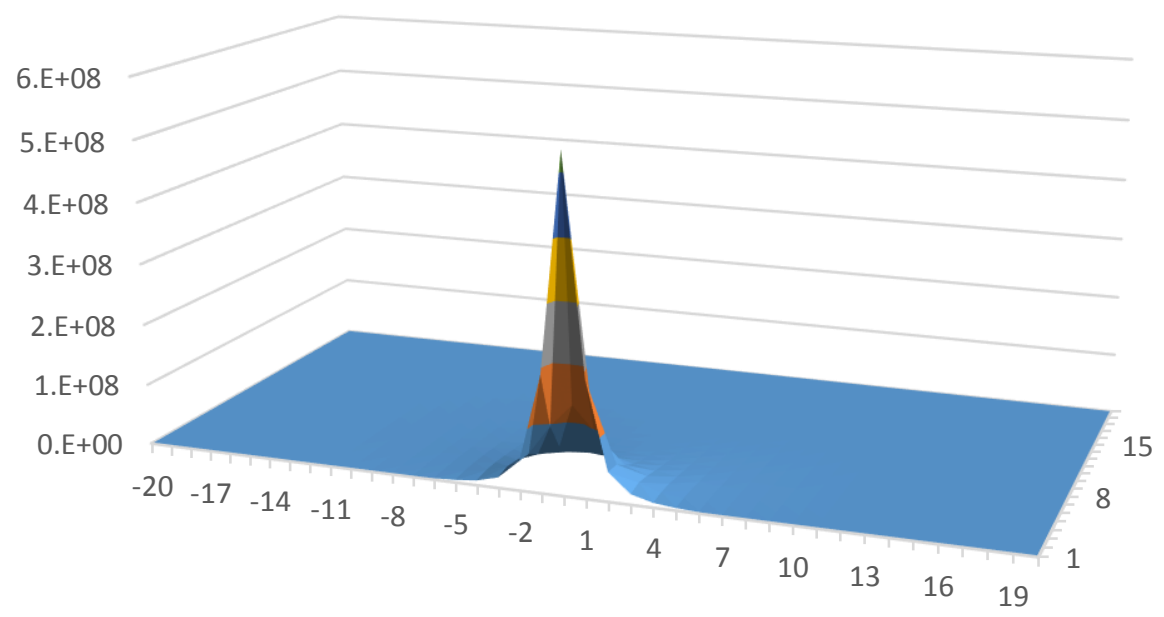

Fig. 4 Magnetic field component of $\mathrm{Br}$ of a monopole. The unit of $\mathrm{Br}$ is [T], while the unit of distance scale of $\mathrm{z}$ $r$ is $10^{-13}[m]$. As shown, the central point, at which a monopole locates, takes the divergence. Note that this distribution is microscopic one of single monopole that is different from macroscopic one.

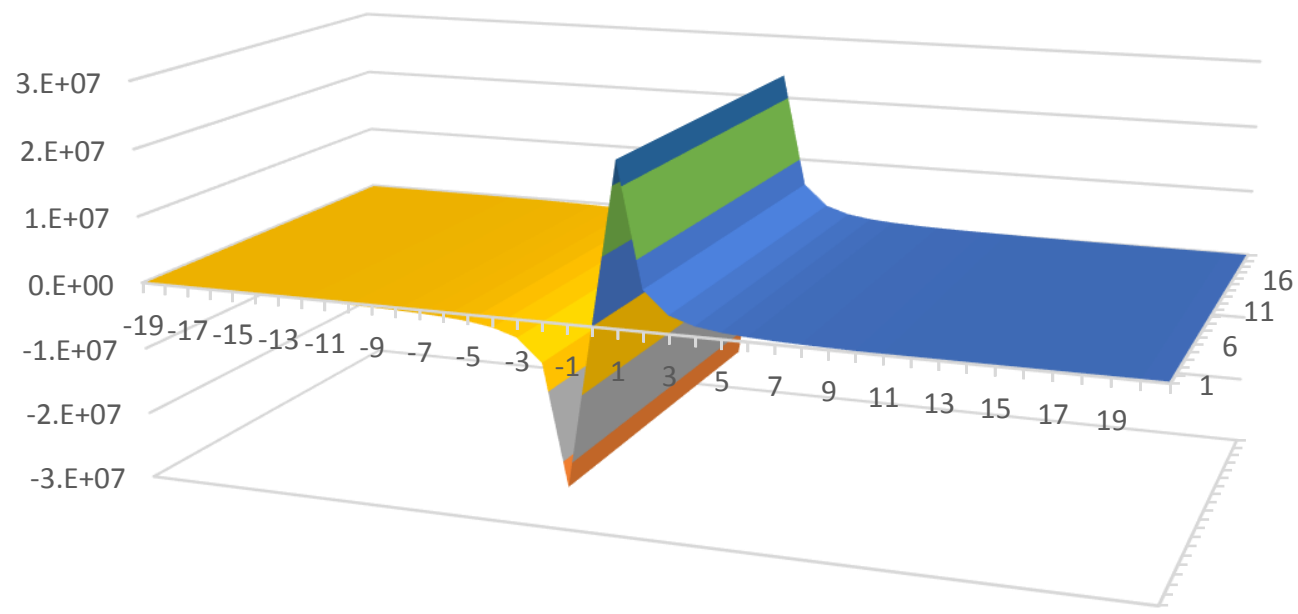

Fig. $5 \mathrm{Bz}$ component of a monopole. Similarly the unit of $B z$ is [T], and the unit of distance of r-z is $10^{-13}[m]$. As shown, the components whose signs are opposite are distributed, which demonstrates the existence of a magnetic monopole. Note that, because this is a $B z$ component not $B r$ component, there is not decay along r-direction. 
Moreover Fig. 6 to Fig. 9, the macroscopic simulation's results are shown. These figures were depicted from the simulating equations derived in Theory section.

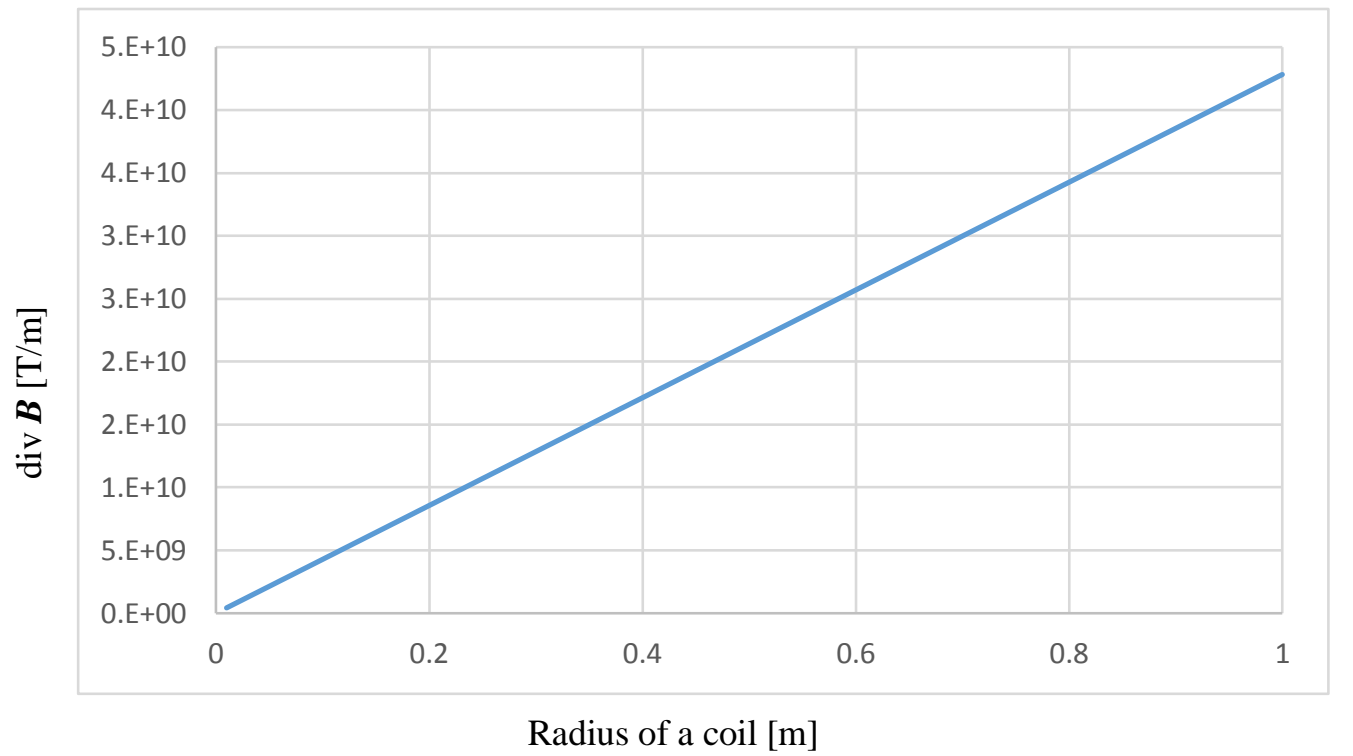

Fig. 6 Dependence of $\operatorname{div} B$ on radius of a coil. As shown, this physical value increases in radius of a coil. Assuming that the Poisson equation can hold, it is possibly considered that the charge density $\rho$ in eq. (11) of $\operatorname{div} B$ increases, depending on radius of a coil.

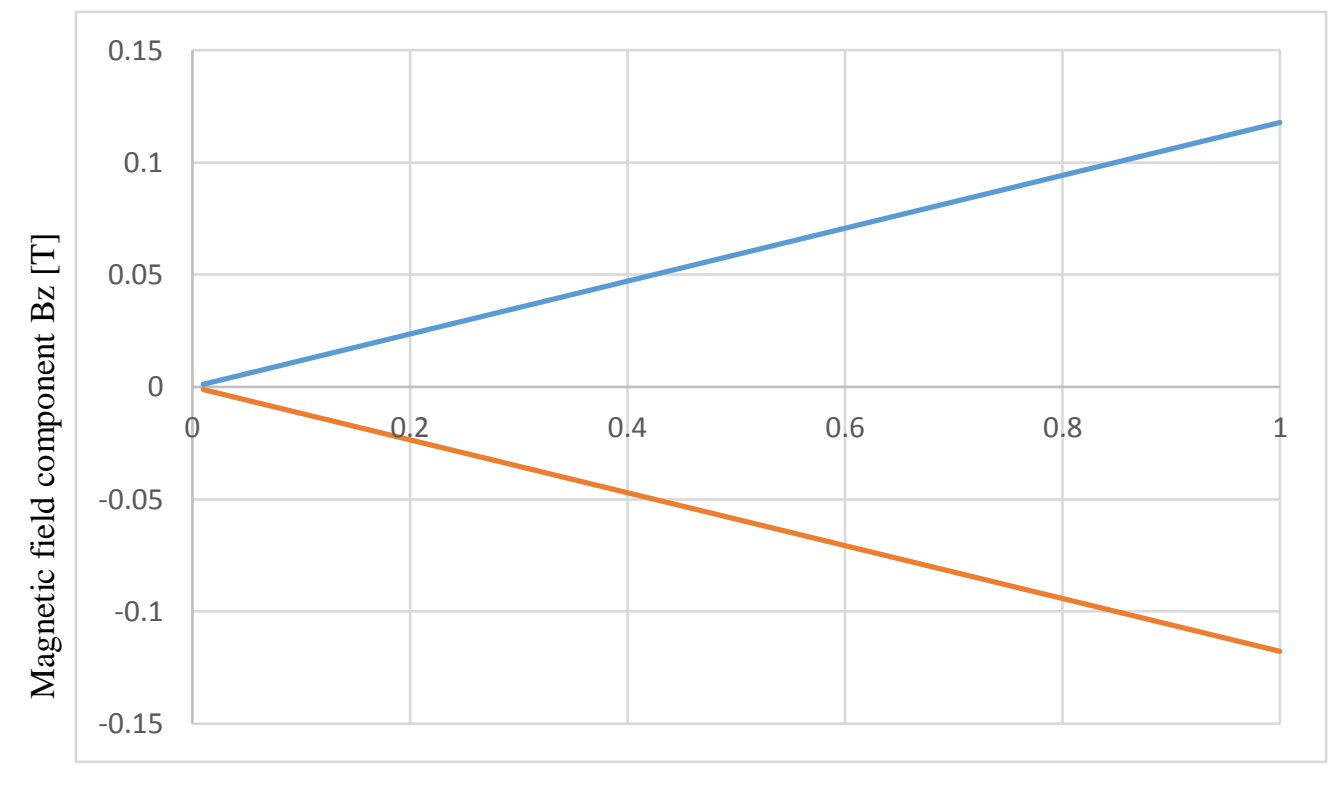

Radius of a coil [m]

Fig. $7 \mathrm{Bz}$ magnetic field component vs. radius of a coil. This result comes from the macroscopic equations, and thus this magnetic field component depends on the macroscopic values. In this figure, the electric potential 
from the condenser of the proposed device is assumed to be 1.0 [V]. As shown, the larger the radius is, the larger magnetic field component. This is because the monopoles' magnetic distribution is determined by Eq. (11) and thus larger radius of a coil would result in making the charge density in Eq. (11) be larger.

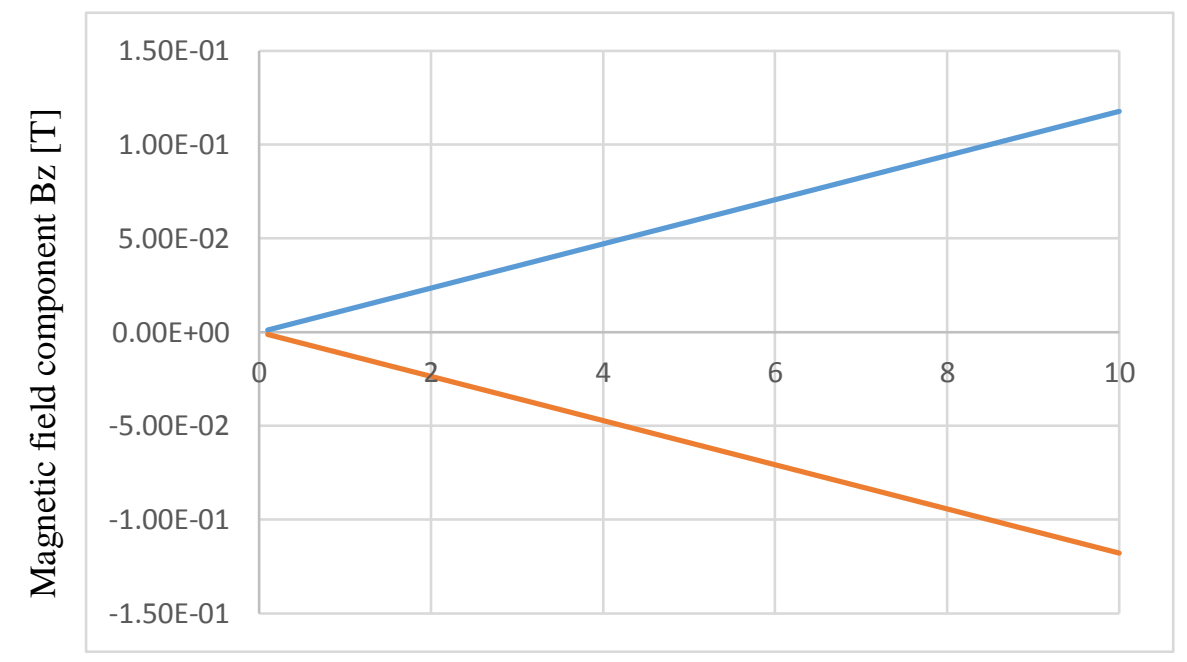

Electric potential of a condenser [V]

Fig. 8 Electric potential of a condenser vs. a magnetic component field $B z$. As mentioned, because this result was derived from the macroscopic equations, this dependence of electric potential is for condenser electric potential. As shown, the magnetic field component $B z$ increases along with electric potentials of a condenser, because the electric potential is, in this paper, directly related to a magnetic potential. (see Eq. (8)) Note that the radius of a coil is kept the constant 0.1 [m].

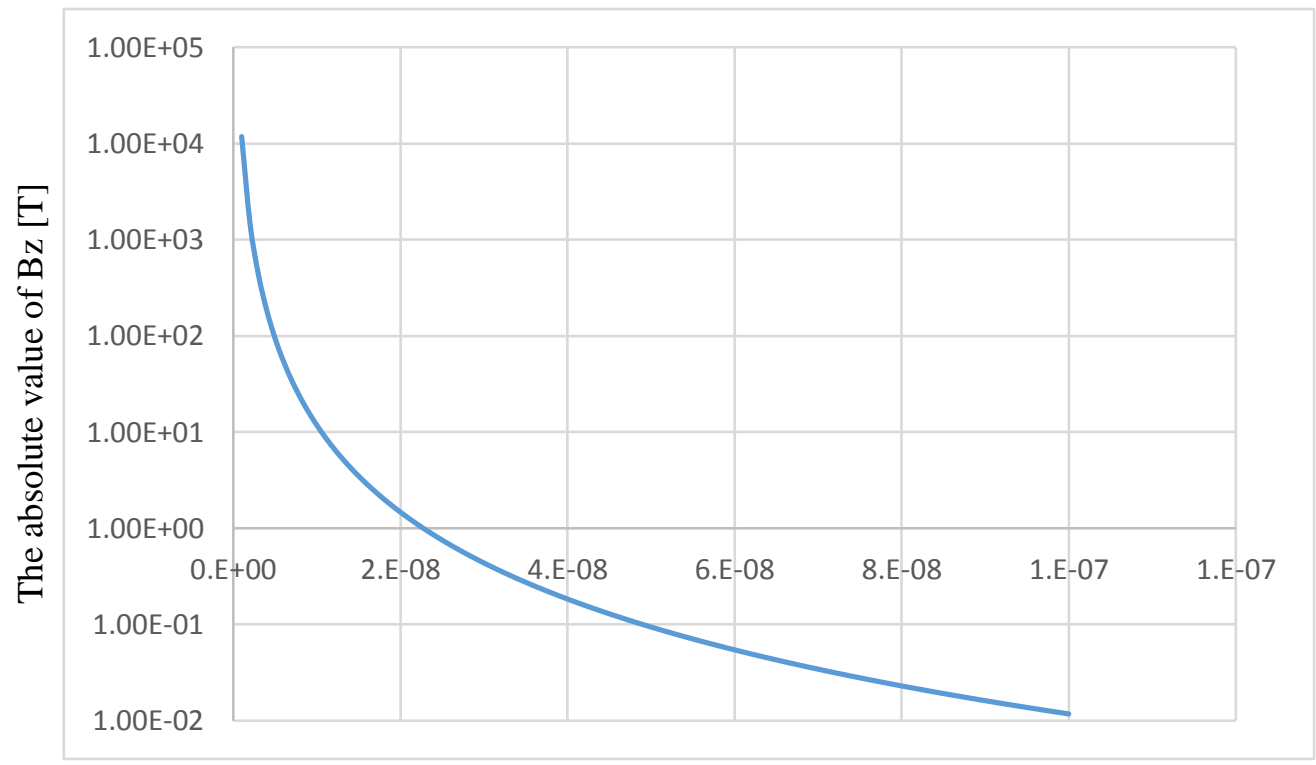

Coherence of a Cooper pair [m] 
Fig. 9 The absolute value of $B z$ magnetic field component vs. superconducting coherence. Note that the perpendicular axis is logarithm. As shown, the magnetic field component $B z$ decreases along with coherence $\xi$. This implies that, the larger superconducting critical temperature is, the larger the magnetic field component Bz.

\section{Discussion}

5.1 Review of a quantized space-time (The zero-point energy and concept of a quantized space-time)

Let us review a concept of space-time which was many times employed in our previous paper [18-20]. The reason of this review is to introduce radius of a monopole and to consider why our universe has only the existing Maxell equations generally.

The concept of quantized space-time, as well as the zero-point energy, can be especially elaborated in a vacuum condition. As such, we begin by describing each concept by solving the Dirac equation. The equation shows that inside a vacuum, during formation of electron and positrons, a mass gap that exists between them can be represented by the relation

$$
\hbar \omega_{0}=2 m_{e} c^{2},
$$

in which $\omega_{0}, m_{e}$, and $c$ are the angular frequency, electron mass, and speed of light, respectively.

Equation (54) can be interpreted in the form

$$
\frac{1}{2} \hbar \omega_{0}=m_{e} c^{2}
$$

where the term in the left-hand side is identical to the expression of zero-point energy based on the Hamiltonian of the harmonic oscillation

$$
\mathrm{H}=\left(\mathrm{n}+\frac{1}{2}\right) \hbar \omega_{0} \text {. }
$$

Note that the first-term in the expanded form of Eq. (56) relates to time-dependent electromagnetic fields, which are calculated in depth in the quantum mechanics, whereas the second term relates to time-independent electromagnetic fields. Indeed, this zero-point energy's expression is related to the static electromagnetic fields. If the angular frequency $\omega_{0}$ is constant, then the zero-point energy should be considered as the universal specific constant energy. Eq. (55) also describes the energy gap of the vacuum based on the result of the Dirac equation. Therefore, the zero-point energy could be taken as an expression of the basic energy of the vacuum. As we have discussed in [19], the mass of an electron is the most basic parameter; thus, Eq. (55) provides a constant quantized space $\lambda_{0}$ and a quantized time $\mathrm{t}_{0}$, which are defined as follows:

$$
\lambda_{0}=\frac{\hbar}{2 m_{e} c},
$$




$$
t_{0}=\frac{\hbar}{2 m_{e} c^{2}}
$$

Consequently, we can derive a more general equation of constant quantized time-space length and time as follows:

$$
\begin{aligned}
& \lambda_{c}=\lambda_{0} \sqrt{1-\frac{v^{2}}{c^{2}}}, \\
& t_{c}=t_{0} \sqrt{1-\frac{v^{2}}{c^{2}}} .
\end{aligned}
$$

Accordingly, we have described in [19] that the gravitational force or Lorentz force from the magnetic fields comes from the fact that up-and down-leptons that are embedded in a quantized space-time $\lambda_{c}$ form rotations and then combine with each other as paired quantized space-time because of the attractive force from the Lorentz force or gravity. This paired quantized space-time behaves like a boson. A schematic representation of this phenomenon is presented in Fig. 10.

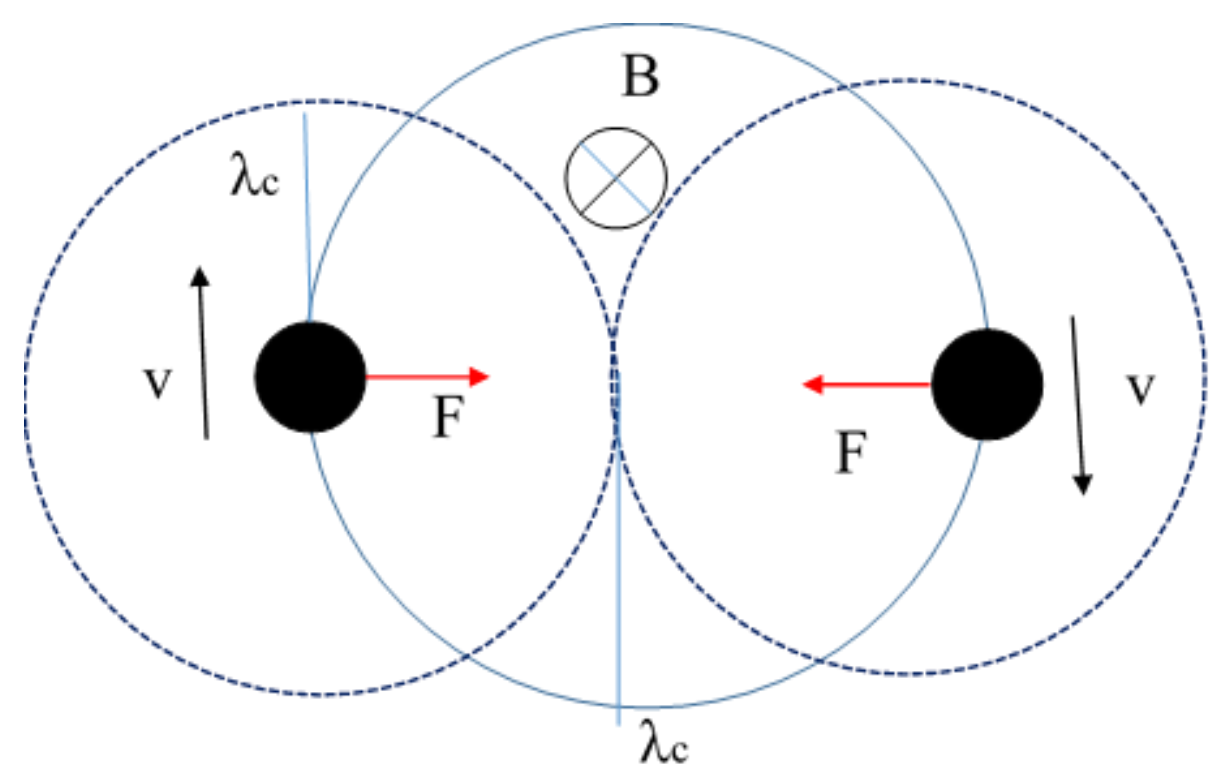

Fig. 10 A schematic model describing a quantized force $\mathbf{F}$. The up- and down-spin electrons do not have real bodies but are embedded in a quantized space. That is, each of the two quantized spaces rotates to create a quantized magnetic field energy, which then produces the Lorentz forces $F$, which are equal to the attractive gravitational force $\mathbf{F}$. 


\subsection{Properties of a monopole particle}

In general, every particle must have a spin angular momentum. However, if a magnetic monopole had a spin angular momentum, that magnetic flux would form a loop. Thus, a monopole must not have a spin angular momentum. This fact can be described as follows:

Remind that our monopole is created by the fact that two Cooper pairs (i.e., four electrons) are combined. Thus, in principle, the following balance between angular momentum and spin angular momentum should be formed in order that the monopole has no spin angular momentum.

$|\vec{l}|=4|\vec{s}|$,

where $\boldsymbol{l}$ and $s$ denote the angular momentum in that two Cooper pairs take their self-rotation and the normal spin angular momentum, respectively.

The left-hand side is calculated as

$l=a \cdot 4 p$,

$p=m v$,

$v=a \omega$,

where $a$ and $\omega$ denote the radius of the self-rotation, and the angular frequency, respectively.

Using the following equation,

$\hbar \omega=2 \mathrm{~m} c^{2}$,

the angular frequency is deleted.

Thus,

$l=a^{2} 4 m \frac{2 m c^{2}}{\hbar}=\frac{8 a^{2} m^{2} c^{2}}{\hbar}$.

Thus Eq. (61) becomes

$\frac{8 a^{2} m^{2} c^{2}}{\hbar}=\frac{\hbar}{2} \times 4$.

As a result,

$\mathrm{a} \approx \frac{\hbar}{2 m c}$.

This is the value of radius of a monopole, which is importantly equal to the quantized space-time in the previous reviewed section. See eq. (57) in the reviewed section.

\subsection{Symmetry of an electric field $\boldsymbol{E}$ and a magnetic field $\boldsymbol{B}$}

This paper described that there are two types of assemblies in terms of Maxwell equations. The crucial point is that the roles between a magnetic field $\boldsymbol{B}$ and an electric field $\boldsymbol{E}$ vectors are completely altered. This implies that originally a magnetic field $\boldsymbol{B}$ and an electric field $\boldsymbol{E}$ are symmetric. Although these 
are symmetric, the reason why we always measure the existing Maxwell equations is based on the quantized space-time structure as reviewed in the previous reviewed section. This implies that the condition our newly proposed Maxwell equations are applied is a rare case and that, to measure our newly proposed Maxwell equations, it is required to prepare the specific setup, which implies that a monopole does not exist in our normal nature and universe but can be created artificially.

\subsection{Interpretation of results of simulations}

The result figures from Fig. 6 to Fig. 9 indicate the macroscopic magnetic field distribution by many monopoles. As shown, besides the $B_{r}$ component, $\pm B_{Z}$ magnetic field components are depicted. As shown in the schematic figure 11, this implies that, as a condition of existences of the monopoles, it is imperative to measure $\pm B_{z}$ magnetic field components as well as $B_{r}$ component.

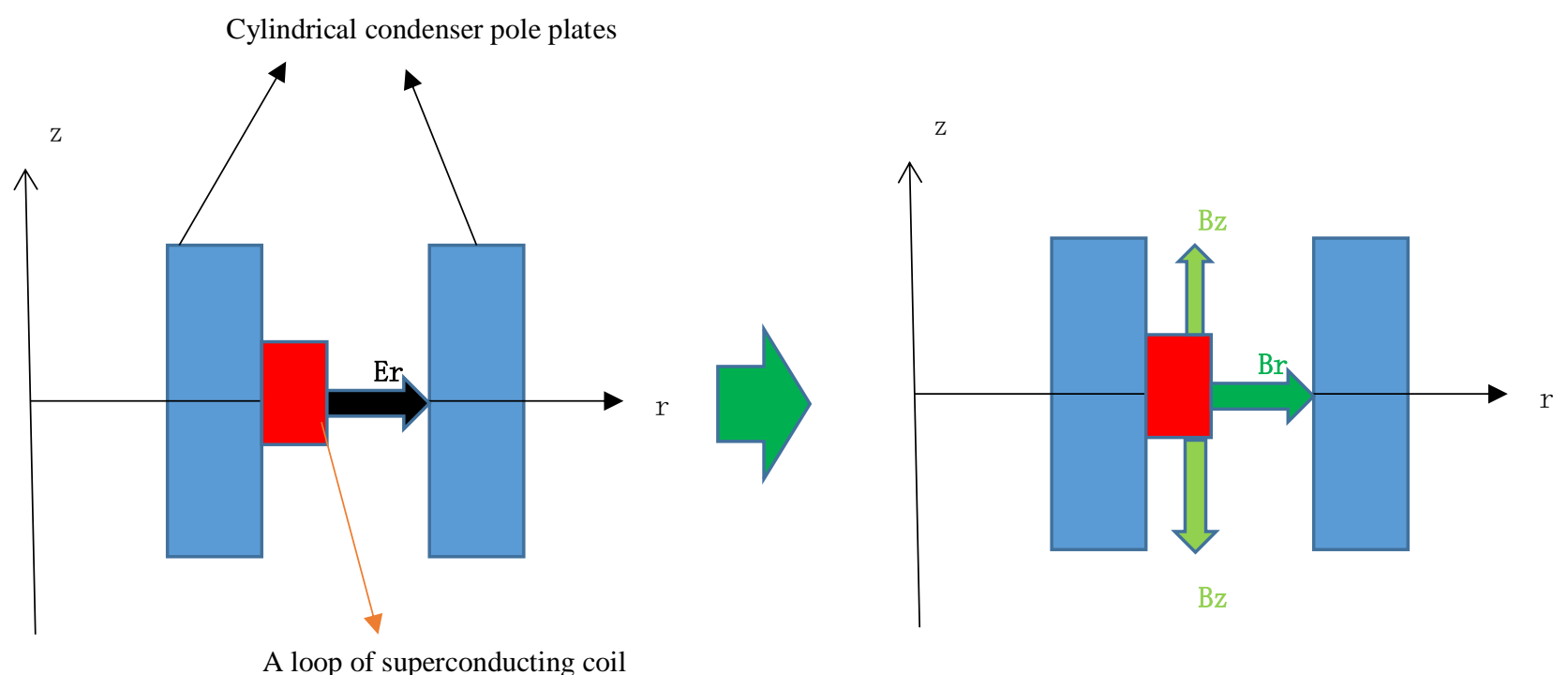

Fig. 11 Schematic of the transition from a normal state to the monopole state. Besides that the electric field component $\mathrm{Er}$ changes to the magnetic field component $\mathrm{Br}$, importantly the magnetic-filed components $\mathrm{Bz}$ appear after the transition. It is imperative to measure these magnetic field components $B z$ in experiments as a follow-up.

\subsection{Significances of the present paper}

It is believed that a natural monopole does not exist. However, this paper described that it is possible to create a magnetic monopole artificially. The significances are that a new elementary particle has 
been predicted and that Maxwell equations are symmetric between an electric field $\boldsymbol{E}$ and a magnetic field $\boldsymbol{B}$. In this paper, these alternative equations were derived as both static ones and time-dependent ones. Although these alternative Maxwell equations exist only under a specific condition, the fact that there are another assembly of Maxwell equations implies that a new insight is provided to electromagnetism field of physics. Moreover, concerning quantum mechanics, we succeeded in describing the wave function that describes a monopole.

\section{Conclusion}

In this paper, we described the potential existence of an artificial magnetic monopole and proposed new electromagnetism equations. That is, we proposed the new method of creating magnetic monopoles. Based on this device and considering the stationery wave and the Lorentz conservations, we obtained the existence of a magnetic monopole. In the process of this theory, the new and alternative Maxwell equations were obtained as well as obtaining the meaning of quantum mechanics of monopoles. Moreover, the numerical calculations assisted them. As a result, we could obtain the divergent distribution of the magnetic fields theoretically.

The measurements of macroscopic divergent magnetic field are simply requirements condition. That is, it is further required to test whether truly a monopole particle is created. As a follow-up, we wish to consult this point with experts of experiments of the condensed matter physics. Moreover, it is important to catch the electromagnetic wave regarding magnetic monopoles as a follow-up.

\section{References}

[1] P.A.M.Dirac, Proc.R.Soc.London,133, 60(1931)

[2] G.’t Hooft, Nucl. Phys., B29, 276 (1974)

[3] K.Sato, Phys.Rev.Lett, 99B, 66 (1981)

[4] C. Castelnovo, et al, arXiv: 0710.5515v2 (2013)

[5] D.J.P. Morris, et al Science 326, 411-414 (2009)

[6] I.Chuang et al, Science 251, 1336-1342 (1991)

[7] Z. Fang, et al Science 302, 92-95 (2003)

[8] P. Milde, et al. Science 340, 1076-1080 (2013)

[9] V. Pietila, et al Phys. Rev. Lett 103, 030401 (2009)

[10] M. W. Ray, et al, Nature 505, 657-660 (2014)

[11] V. D. Barger and M.G. Olsson, "Classical Electricity and Magnetism” published by Allyn and Bacon, Inc., Newton, Massachusetts 02159 (Japanese Edition) p. 177 (2006)

[12] W. H. Hayt, “Engineering Electromagnetics $5^{\text {th }}$ edition”, p.182, p. 185 (1999) 
[13] R. B. Laughlin, “A Different Universe Reinventing Physics from the Bottom Down" (Japanese version) pp.46-pp.57, Nikkei BP Sha in Tokyo (2006)

[14] N. Kanazawa, et al, Nature communications 7, 11622 (2016)

[15] Xiang-Fa Zhou, et al, Phys. Rev. Lett. 120, 130402 (2018)

[16] K. Tiurev, et al arXiv 1810.03725 (2018)

[17] S. Koide, “Quantum theory”, pp. 63-pp.68, Shokabou in Tokyo (1997)

[18] S. Ishiguri, "Unified Field Theory for Electromagnetic and Gravity Fields with the Introduction of Quantized Space-Time and Zero-point Energy", Preprints 2020, 2020070462 (doi:10.20944/preprints202007.0462.v1)

[19] S. Ishiguri, "A Unified Theory of All the Fields in Elementary Particle Physics Derived Solely from the Zero-point Energy in Quantized Spacetime", Preprints 2019, 2019070326 (doi: 10.20944/preprints201907.0326.v1)

[20] S. Ishiguri, "Theory on Neutrino Self-Energy and Neutrino Oscillation with Consideration of Superconducting Energy Gap and Fermi's Golden Rule", Preprints 2019, 2019100080 (doi: 10.20944/preprints201910.0080.v1)

\section{Additional information}

This paper is not related to any competing interests such as funding, employment and personal financial interesting. Moreover, this paper is not related to non-financial competing interesting

\section{Acknowledgement}

1) We sincerely appreciate MDPI for presenting the 1st version of this paper;

S. Ishiguri, "Theoretical Studies on the Creation of Artificial Magnetic Monopoles", Preprints 2018, 201820194 (doi; 10.20944/preprints201812.0194.v1)

2) We thank Enago (www.enago.jp) for English language Review. 